\title{
Measurement of microRNA with isothermal DNA amplification on fully automated immunoassay analyzers
}

\author{
Makoto Komori $^{1} \cdot$ Ken Komiya ${ }^{2} \cdot$ Takuma Shirakawa $^{1} \cdot$ Takamitsu J. Morikawa $^{1} \cdot$ Toru Yoshimura $^{1}$
}

Received: 4 March 2019 / Revised: 15 April 2019 / Accepted: 26 April 2019 / Published online: 3 June 2019

(C) The Author(s) 2019

\begin{abstract}
MicroRNAs (miRNAs) in a blood sample are usually measured by quantitative reverse transcription PCR (qRT-PCR), microarray, and next-generation sequencing (NGS) which requires time-consuming pre-treatment, manual operation, and a stand-alone instrument. To overcome these disadvantages, miRNA testing has been developed using the automated analyzers routinely used in clinical laboratories. An isothermal DNA amplification reaction was adapted to a fully automated immunoassay analyzer that conducts extraction, amplification, and detection processes at $37^{\circ} \mathrm{C}$ in $44 \mathrm{~min}$. In a reaction vessel, a pre-designed single-stranded signal DNA was amplified in the presence of miRNA, using DNA templates, DNA polymerase, and nicking endonuclease. Then, the amplified signal DNA was hybridized by one DNA probe attached to a magnetic particle and another DNA probe labeled with acridinium ester. After the chemiluminescence reaction, luminescence intensity was automatically measured. The automated assays of cancer-related miRNAs were implemented on the analyzer with throughput of 66 tests per hour. In the assays with onestep amplification, three miRNAs (miR-21-5p, miR-18a-5p, and miR-500a-3p) at concentrations lower than $100 \mathrm{fM}$ were automatically detected and the cross reactivity for miR-21-5p with fifteen similar miRNAs was not higher than $0.02 \%$. In the assay with two-step amplification, detection sensitivity and amplification rate for miR-21-5p were $3 \mathrm{fM}$ and 103-fold, respectively. The coefficient of variations (CVs) in the measurement at the target concentrations from $5 \mathrm{fM}$ to $1000 \mathrm{pM}$ were less than $8 \%$. Furthermore, we also achieved automated nucleic acid detection in human serum. The proposed fully automated miRNA assays showed high sensitivity, low cross reactivity, and reproducibility suitable for clinical use.
\end{abstract}

Keywords MicroRNA measurement · Isothermal DNA amplification · Automated immunoassay analyzer · Cancer diagnostic marker $\cdot$ Chemiluminescence microparticle assay

\section{Introduction}

MicroRNAs (miRNAs) are functional small non-coding RNAs of 19-24 nucleotides that are involved in regulating many biological processes, including proliferation,

Makoto Komori and Ken Komiya contributed equally to this work.

Electronic supplementary material The online version of this article (https://doi.org/10.1007/s00216-019-01878-z) contains supplementary material, which is available to authorized users.

Makoto Komori

makoto.komori@abbott.com

1 Research and Development, Abbott Japan Co. Ltd, 278 Matsuhidai, Matsudo, Chiba 270-2214, Japan

2 School of Computing, Tokyo Institute of Technology, J2-51, 4259, Nagatsuta-cho, Midori-ku, Yokohama, Kanagawa 226-8503, Japan differentiation, apoptosis, and development $[1,2]$. The aberrant expression of miRNAs is closely related to cancer [3-6]. The miRNAs are stable in blood because most of miRNAs are bound to protective proteins such as Argonaute complexes and some miRNAs are inside or outside membrane vesicles such as exosomes [7-10]. Recently, circulating miRNAs have attracted attention as new potential biomarkers for cancer diagnosis, prognosis, and detection of recurrence [11-16]. However, the measurement of miRNAs is still challenging because miRNAs have cumbersome characteristics: short length (19-24 nucleotides), low concentration, and sequence homology among the miRNA family [17]. Research activities have been conducted to develop a miRNA quantification method for practical use [18-21].

The current major measurement methods of miRNAs are quantitative reverse transcription PCR (qRT-PCR), microarray, and next-generation sequencing (NGS) [2, 22]. While both microarray and NGS are suitable for screening and 
discovery purposes, qRT-PCR is still the first choice for validation and clinical tests with a high number of samples [16]. The qRT-PCR has been preferably used due to its high sensitivity and high specificity [2], but qRT-PCR in clinical laboratories has several disadvantages. First, a large qRT-PCR instrument used only for detecting nucleic acids is needed $[23,24]$. Second, measurement cost is high due to the expensive instrument and the manual operation required for extraction and purification processes $[25,26]$. Third, throughput is decreased by the long purification process due to blood coagulation under high-temperature conditions [26]. These disadvantages of qRT-PCR make it difficult to introduce miRNAs into clinical laboratories as cancer diagnostic markers [27, 28]. In addition, the manual operation has risk of human error.

Fully automated immunoassay analyzers are widely used in clinical laboratories to measure various proteins and chemical compounds as diagnostic markers in biological samples including serum, plasma, and urine [29-31]. In most fully automated immunoassay analyzers, luminescence intensity of chemiluminescent substrates is detected, such as acridinium ester, ruthenium complex, and luminol, after the labeled antibodies or antigens bind to the analytes [32]. The existing fully automated immunoassay analyzers can be used to process and measure miRNAs in serum under physiological temperature conditions. This avoids blood coagulation and the long purification process, eliminates the need to perform manual extraction processes, and eliminates the need to transfer the sample to the stand-alone qRT-PCR instrument. Implementation of miRNA testing on the automated immunoassay analyzers will lead to shorter test time and cost reduction and thus is significantly advantageous both for medical facilities and patients $[27,28]$.

According to the meta-analysis of clinical studies about miR-21-5p as a gastric cancer marker in serum and plasma, the cutoff concentrations of miR-21-5p were $37.3 \mathrm{fM}, 50 \mathrm{fM}$, and $59.5 \mathrm{fM}$ at three sites [13]. Therefore, a miR-21-5p assay needs a detection sensitivity around $50 \mathrm{fM}$. A previous study reported a detection sensitivity of miRNA at $1 \mathrm{pM}$ using an acridinium-labeled antibody to a heterohybrid of miRNA and DNA on a fully automated immunoassay analyzer without DNA amplification [27]. To establish a more sensitive miRNA measurement, development of a method for DNA amplification is needed. However, the clinically approved methods of DNA amplification, such as loop-mediated isothermal amplification (LAMP) and strand displacement amplification (SDA), require the high reaction temperature at $60^{\circ} \mathrm{C}$ and $54^{\circ} \mathrm{C}$, respectively $[33,34]$. Although rolling circle amplification (RCA) achieves DNA amplification at 30$37^{\circ} \mathrm{C}$, it requires several hours for ligation reaction of padlock probe before DNA amplification $[35,36]$.

We recently proposed a novel isothermal DNA amplification method, low-temperature amplification (L-TEAM), and demonstrated leak-free amplification [37]. In this method, pre- designed signal DNA is amplified exponentially in the presence of a target nucleic acid at constant $37{ }^{\circ} \mathrm{C}$ with the use of two DNA templates. In the present study, we adapted the miRNA measurement via L-TEAM for use with a fully automated immunoassay analyzer.

The exponential DNA amplification using immunoassay analyzers could lead to false positive results because the automated immunoassay analyzers are open systems. There is a possibility that the amplified signal sequence may contaminate other reaction vessels via a pipette or aerosol in the analyzer. In the reaction design of exponential amplification including PCR and L-TEAM, in which the end product as an amplified signal triggers amplification of the end product itself, contamination severely interferes amplification results. To minimize potential false positive results due to contamination, we modified L-TEAM to a cascade reaction that consists only of linear amplification. In the cascade reaction, successive sequence conversion of the generated single-stranded DNAs (ssDNAs) reduces the risk of false positive results by avoiding self-triggered exponential amplification and achieves higher amplification rates in comparison to onestep linear amplification.

In this paper, we first describe the automated assays for three miRNAs, i.e., miR-21-5p, miR-18a-5p, and miR-500a$3 p$, that were reported as cancer diagnostic markers $[11,12$, 14], implemented on a fully automated immunoassay analyzer with one-step amplification reaction. Then, we report the assay performances upon the detection of miR-21-5p and miR200 families that were reported as colorectal cancer markers $[38,39]$ on the analyzer with two-step amplification reaction.

\section{Materials and methods}

\section{Materials for amplification and detection}

All oligodeoxyribonucleotides and oligoribonucleotides were synthesized by Gene Design (Ibaraki, Japan). The Converter DNA and Cascade DNA were purified via ion exchange HPLC. The sequences of oligodeoxyribonucleotides and oligoribonucleotides and the chemical modification are described in Electronic Supplementary Material (ESM) Table S1. Bst DNA Polymerase, Large Fragment, Nb.BbvCI, NEB Buffer 2 (final concentration of $10 \mathrm{mM}$ Tris- $\mathrm{HCl}, 50 \mathrm{mM} \mathrm{NaCl}, 10 \mathrm{mM} \mathrm{MgCl} 2,1 \mathrm{mM}$ DTT, $0.1 \%$ Tween 20, pH 7.9), and dNTPs were purchased from New England Biolabs Japan (Tokyo, Japan). Streptavidin-coated magnetic particle, Dynabeads ${ }^{\circledR}$ M-270 Streptavidin was purchased from Thermo Fisher Scientific K. K. (Kanagawa, Japan). The magnetic particles are uniform in size, having a diameter of $2.8 \mu \mathrm{m}$ (https://www.thermofisher.com/jp/ja/ home/brands/product-brand/dynal/streptavidin-coupleddynabeads.html). 


\section{Scheme of signal DNA amplification}

The isothermal one-step signal DNA amplification (onestep amplification) reaction for miRNA detection requires one DNA template called Converter DNA, DNA polymerase having strand displacement activity, nicking endonuclease, dNTPs, and a target miRNA. Converter DNA comprises Signal DNA1 generation sequence, nicking endonuclease recognition sequence (NERS), cover sequence 1 (CS1) which is complementary to Signal DNA1 generation sequence, and target binding sequence (TBS) which is complementary to a target miRNA in the $5^{\prime}$ to $3^{\prime}$ direction (Fig. 1a). At the reaction temperature, Converter DNA forms a hairpin structure comprising an 18-bp stem and avoids binding of Signal DNA1 released from a Converter DNA to the Signal DNA1 generation sequence of another Converter DNA.

When miRNA hybridizes with the TBS of Converter DNA, DNA extension occurs from the $3^{\prime}$ terminal of miRNA by DNA polymerase (steps $i$ and ii in Fig. 1b). During DNA extension, the hairpin structure is opened due to the strand displacement activity of DNA Polymerase, and the DNA strand complementary to Converter DNA is generated (step iii). Then, nicking endonuclease recognizes its recognition sequence in the double-stranded form. Upon nicking reaction, Signal DNA1 is generated (step iv). After that, DNA extension occurs again from the $3^{\prime}$ terminal of nicked position. As a consequence, the formerly generated Signal DNA1 is released because of the strand displacement activity of DNA Polymerase (step v). These polymerization and nicking reactions repetitively occur and linearly amplify Signal DNA1, only in the presence of miRNA without the needs of reverse transcription reaction and tag-adding reaction for generating primer-binding sequence that are required in qRT-PCR.

The isothermal two-step cascade signal DNA amplification (two-step amplification) reaction is also achieved by layering two consecutive linear signal DNA amplification reactions (Fig. 1b, c). In the second step reaction, hybridization of Signal DNA1 with the Signal DNA1 binding sequence of Cascade DNA further triggers the next linear amplification of Signal DNA2, whose sequence is different from that of Signal DNA1, via the repetitive polymerization and nicking reaction similarly to the first step reaction.

\section{Preparation of Capture DNA probe attached to magnetic particles}

Capture DNA probe (13 nucleotides (nt) in length) labeled with biotin-triethylene glycol spacer at its $3^{\prime}$ end was incubated with streptavidin-coated magnetic particles for
$30 \mathrm{~min}$ at a room temperature. After washing the magnetic particles under magnetic attraction, a $0.05 \%(w / v)$ particle solution was prepared.

\section{Preparation of Chemiluminescence DNA probe}

Chemiluminescence DNA probe $(10 \mathrm{nt})$ modified with amino group-six-carbon spacer at its $5^{\prime}$ end was reacted with NHS-conjugated acridinium ester provided by Abbott Laboratories (IL, USA) for overnight at room temperature. The resulting Chemiluminescence DNA probe labeled with acridinium ester was separated by reversed-phase HPLC (Nihon Waters K.K., Tokyo, Japan) and diluted to $100 \mathrm{nM}$.

\section{Automated miRNA detection on the analyzer}

A fully automated immunoassay analyzer, ARCHITECT i system (Abbott Japan, Tokyo, Japan) is used for measuring proteins and chemical compounds in serum, plasma, and urine as diagnostic markers in about $30 \mathrm{~min}$ at clinical laboratories [29]. In the present study, automated miRNA assays were implemented on the immunoassay analyzer as follows. One sample solution and six operational solutions were placed on the analyzer, and the miRNA assay was started. Initially, a $40-\mu \mathrm{L}$ sample solution containing synthetic miRNA in $10 \mathrm{mM}$ Tris- $\mathrm{HCl}$ and $0.01 \%$ BSA ( $\mathrm{pH} 8.0)$ was dispensed into a reaction vessel. Then, a $50-\mu \mathrm{L}$ solution containing detergent was mixed for $7 \mathrm{~min}$ at $37^{\circ} \mathrm{C}$ on the fully automated analyzer. The role of detergent is to liberate miRNAs when the sample is serum. A $30-\mu \mathrm{L}$ solution of this mixture was transferred to another reaction vessel. Next, a $40-\mu \mathrm{L}$ solution of Converter DNA, a $40-\mu \mathrm{L}$ pre-mixed solution of Bst DNA Polymerase, Large Fragment, and $\mathrm{Nb} . \mathrm{BbvCl}$, and a $20-\mu \mathrm{L}$ dNTP solution were dispensed into the reaction vessel and incubated for $7 \mathrm{~min}$ at $37{ }^{\circ} \mathrm{C}$. In this amplification reaction mixture containing Bst DNA Polymerase, Large Fragment, and Nb.BbvCI at 0.07 and 0.09 units $/ \mu \mathrm{L}$, respectively, hybridization of target miRNA to Converter DNA primes DNA extension and Signal DNA1 is linearly amplified by the repetitive polymerization and nicking reaction. After $7 \mathrm{~min}, 70-\mu \mathrm{L}$ of this reaction mixture was transferred to the other reaction vessel. A $50-\mu \mathrm{L}$ solution of Capture DNA probe attached to the magnetic particle was dispensed in the reaction vessel and incubated for $18 \mathrm{~min}$ at $37^{\circ} \mathrm{C}$. In this amplification and capturing reaction mixture containing Bst DNA Polymerase, Large Fragment, and $\mathrm{Nb}$.BbvCI at 0.04 and 0.05 units $/ \mu \mathrm{L}$, respectively, amplification of Signal DNA1 continues and hybridization of Signal DNA1 to a Capture DNA probe concurrently occurs (Fig. 2). After washing the magnetic particles under 
Fig. 1 The schematic illustrations of $\mathbf{a}$ the sequence domains of Converter DNA and Cascade DNA, b linear signal DNA amplification reaction in the onestep amplification assay or the first step of the two-step amplification assay, and $\mathbf{c}$ linear signal DNA amplification reaction in the second step of the two-step amplification assay. Red vertical lines represent base pairings a Converter DNA

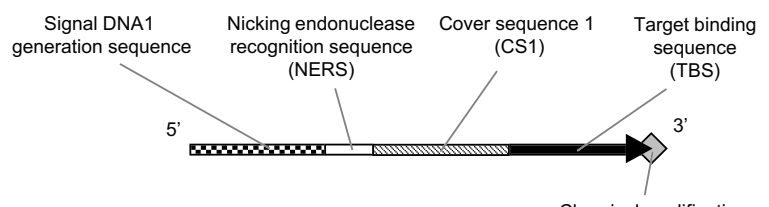

Cascade DNA

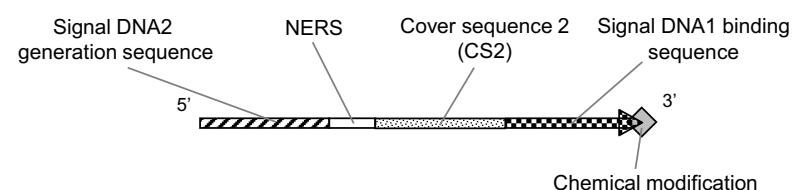

b

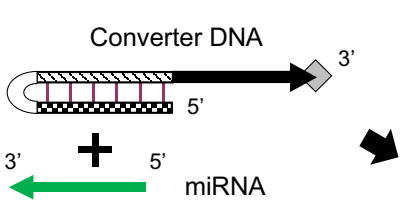

ii)

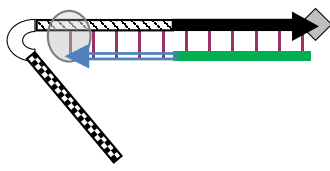

4

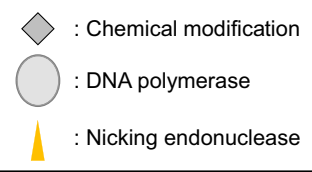

i)

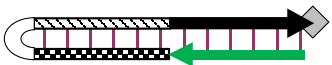

iv)
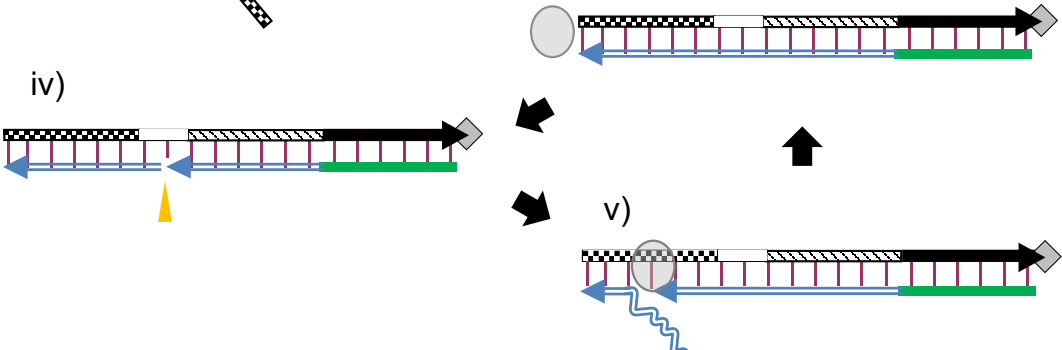

Signal DNA1

v v)

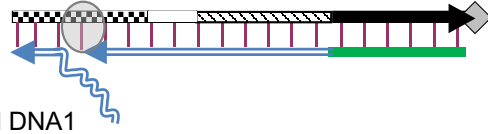

C
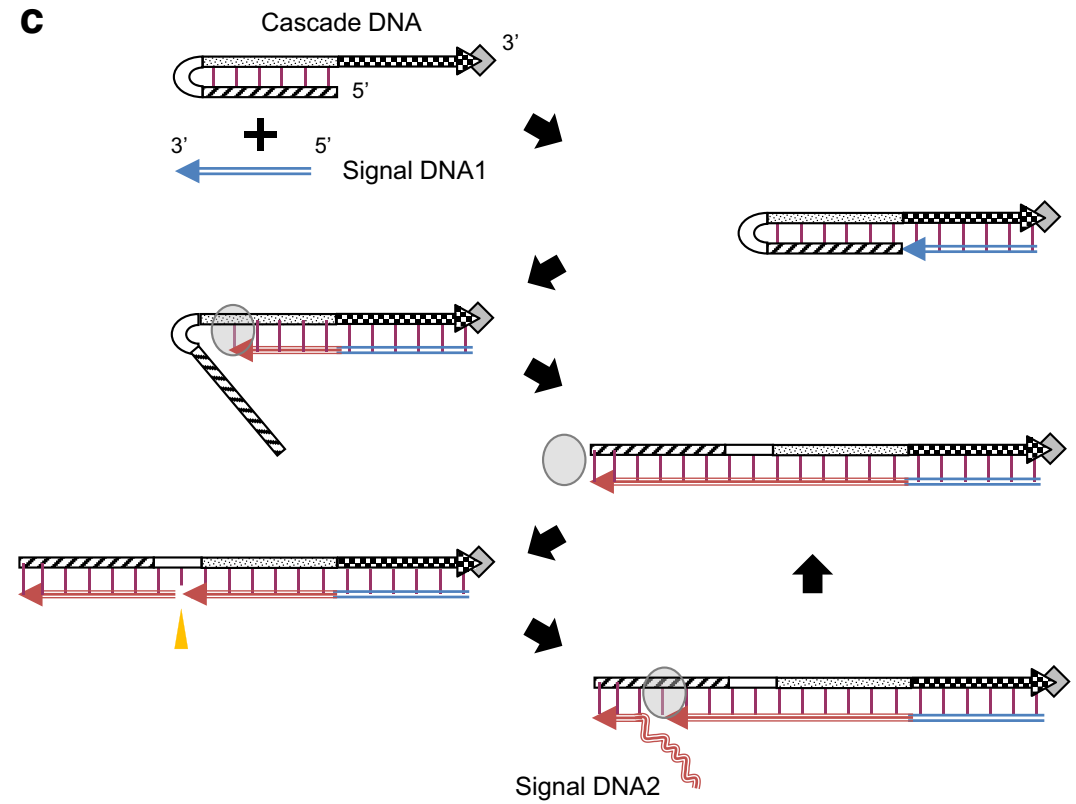
magnetic attraction, a $50-\mu \mathrm{L}$ solution of acridiniumlabeled Chemiluminescence DNA probe was dispensed and incubated for $4 \mathrm{~min}$ at $37^{\circ} \mathrm{C}$. In this hybridization reaction mixture, Chemiluminescence DNA probe hybridizes to Signal DNA1 captured by Capture DNA probe on the magnetic particle. After washing the magnetic particles again under magnetic attraction, pretrigger solution containing hydrogen peroxide and trigger solution containing sodium hydroxide of ARCHITECT $\mathrm{i}$ system (Abbott Japan) were dispensed and incubated for $18 \mathrm{~s}$ at $37{ }^{\circ} \mathrm{C}$. The chemiluminescence of acridinium at the wavelengths in the range of 400 to $500 \mathrm{~nm}$ was measured by the optical system of the analyzer. In principle, the intensity of chemiluminescence is proportional to the amount of amplified Signal DNA1. In the one-step amplification assays, Converter DNA-21, Converter DNA-18, and Converter DNA-500, which were designed for the corresponding target miRNAs (miR-21-5p, miR$18 \mathrm{a}-5 \mathrm{p}$, and miR-500a-3p), were used. The final concentrations of Converter DNA and each dNTP were $1.4 \mathrm{nM}$ and $100 \mu \mathrm{M}$, respectively. In the two-step amplification assays for miR-21-5p and miR-200 family, the reaction was performed similarly as the above described with the Converter DNAs, each having the TBS for the corresponding target miRNA (miR-21-5p, miR-200a, miR200b, and miR-200c), except for the final concentrations of Cascade DNA and each dNTP were $4.2 \mathrm{nM}$ and $10 \mu \mathrm{M}$, respectively.

\section{Automated nucleic acid detection in human serum on the analyzer}

The ssDNA having the sequence the same as miR-21-5p, termed miD-21-5p, was spiked directly into normal human serum. The sample was measured via the two-step amplification assay for miR-21-5p.

\section{Results and discussion}

\section{Automated miRNA detection with one-step amplification on the analyzer}

We measured different concentrations of synthetic miR-21-5p from 0.01 to $1000 \mathrm{pM}$ in triplicate, and $0 \mathrm{pM}$ in replicates of 5 via the one-step amplification assay for miR-21-5p using Converter DNA-21. The Converter DNA was designed to form a hairpin structure by the introduction of cover sequence as shown in Fig. 1. The hairpin structure accelerates DNA amplification by preventing signal DNA released into the solution from hybridizing to signal generation sequence of intact Converter DNA (see ESM Methods, ESM Results, ESM Fig. $\mathrm{S} 1$, Table S2). The assay results were obtained in $44 \mathrm{~min}$ after initial sample dispense with throughput of 66 tests per hour. The resulting dose-response curve of the miR-21-5p assay is shown in Fig. 3a. The coefficient of variations (CVs) of chemiluminescence intensity (CI) for the target at concentrations of $1 \mathrm{pM}$ and higher were less than $6 \%$. The CVs of CI even at low target concentrations both of 10 and $100 \mathrm{fM}$ were $10 \%$, and that of the background $\mathrm{CI}$ at $0 \mathrm{pM}$ was $13 \%$. The detection sensitivity was in the range from 10 to $100 \mathrm{fM}$. Similarly, we also measured miR-18a-5p and miR-500a-3p on the analyzer with Converter DNA-18 and Converter DNA-500, respectively (Fig. 3a). For clarity, each doseresponse curve was separately shown in ESM Fig. S2A-C.

The one-step amplification assays for miR-18a-5p and miR-500a-3p, which were developed by changing the TBS of Converter DNA-21, exhibited comparable sensitivity as

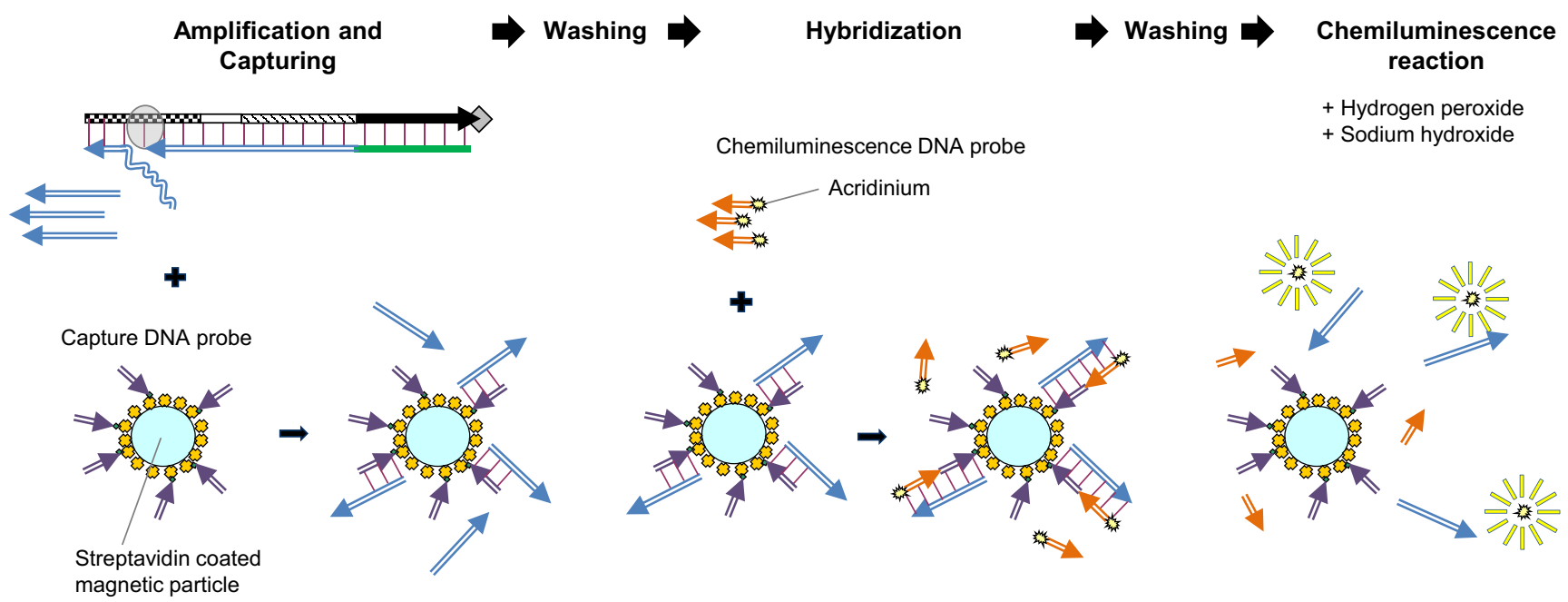

Fig. 2 The schematic illustrations of capturing of signal DNA followed by chemiluminescence reaction on the immunoassay analyzer 
Fig. 3 a Dose responses in the respective one-step amplification assays for miR-21-5p, miR-18a$5 \mathrm{p}$, and miR-500a- $3 \mathrm{p}$ on the analyzer. The bar shows \pm 2 standard deviations. Each sample was measured in triplicate except for the blank sample (replicate (rep.) $=5$ ). $\mathbf{b}$ Cross reactivity test of fifteen human miRNAs which have sequences similar to that of miR$21-5 p$ in the one-step amplification assay for miR-21-5p on the analyzer. The bar shows \pm 2 standard deviations. Each miRNA sample was measured in triplicate except for the blank sample (rep. $=5$ )

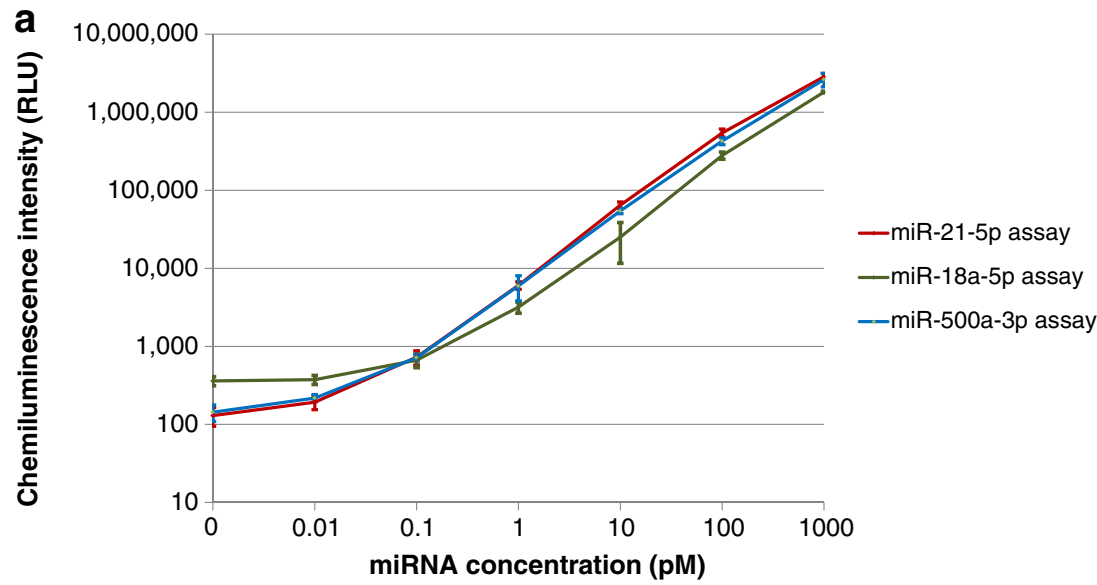

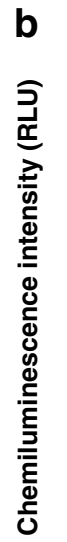

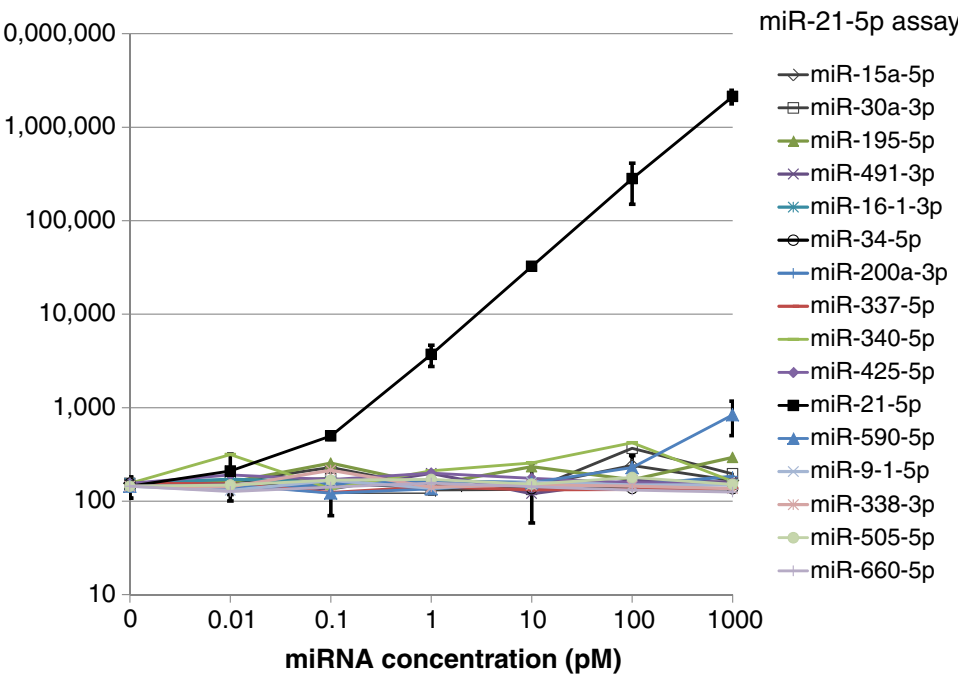

shown in the dose-response curves (Fig. 3a). In the present assay system, the target miRNA is easily altered only by changing the TBS of Converter DNA since miRNA is converted to the common signal DNA to be detected with the common Capture DNA probe and Chemiluminescence DNA probe. More than a hundred circulating miRNAs in blood have been so far identified as diagnostic, prognostic, or predictive biomarkers for different types of cancers [15]. The proposed assay allows the use of a common detection system and promotes the development of multiple miRNA assays.

Next, we investigated the cross reactivity of the one-step amplification assay for miR-21-5p to evaluate the sequence specificity. We selected fifteen human miRNAs which have sequences similar to that of miR-21-5p according to the miRNA database, miRBase [2, 40]. The numbers of bases identical to those in miR-21-5p sequence ranged from 8 to 14 (ESM Table S3). We measured the samples of each synthetic miRNA at the concentrations from 0 to $1000 \mathrm{pM}$. Concentrations of synthetic miRNAs which interfered with the assay of miR-21-5p were estimated by the dose-response curve of miR-21-5p. The concentration was defined as interfering miR-21-5p concentration (Fig. 3b). The cross reactivity was evaluated with the ratio of the interfering miR-21$5 \mathrm{p}$ concentration to each synthetic miRNA concentration. No cross reactivity was observed for fourteen miRNAs (miR-15a5p, miR-30a-3p, miR-195-5p, miR-491-3p, miR-16-1-3p, miR-34a-5p, miR-200a-3p, miR-337-5p, miR-340-5p, miR425-5p, miR-9-1-5p, miR-338-3p, miR-505-5p, miR-660$5 p)$ (Fig. 3b). At the highest, $0.02 \%$ of cross reactivity was found in miR-590-5p at the concentration of $1000 \mathrm{pM}$, which has 13 bases identical to those in miR-21-5p sequence. These results indicated high sequence specificity of the proposed miR-21-5p assay.

\section{Automated miR-21-5p detection with two-step amplification on the analyzer}

The detection sensitivity of the one-step amplification assay for miR-21-5p in the range from 10 to $100 \mathrm{fM}$ was not sufficient because the cutoff concentration of miR-21-5p in serum and plasma for cancer diagnosis is around $50 \mathrm{fM}$ [13]. We extended the one-step amplification assay to the 
Fig. 4 a Dose response in the two-step amplification assay for miR-21-5p at the concentrations from 0 to $1000 \mathrm{pM}$ on the analyzer. The bar shows \pm 2 standard deviations. Each sample was measured in replicates of $5 . \mathbf{b}$ Dose response for the detection sensitivity test in the two-step amplification assay for miR-21$5 \mathrm{p}$ at the concentrations from 0 to $50 \mathrm{pM}$ on the analyzer. The bar shows \pm 2 standard deviations. Each sample was measured in replicates of 20. c Cross reactivity test of precursor miR-21-5p in the two-step amplification assay for miR-21-5p on the analyzer. The bar shows \pm 2 standard deviations. Each sample was measured in triplicate except for the blank sample (rep. $=5)$

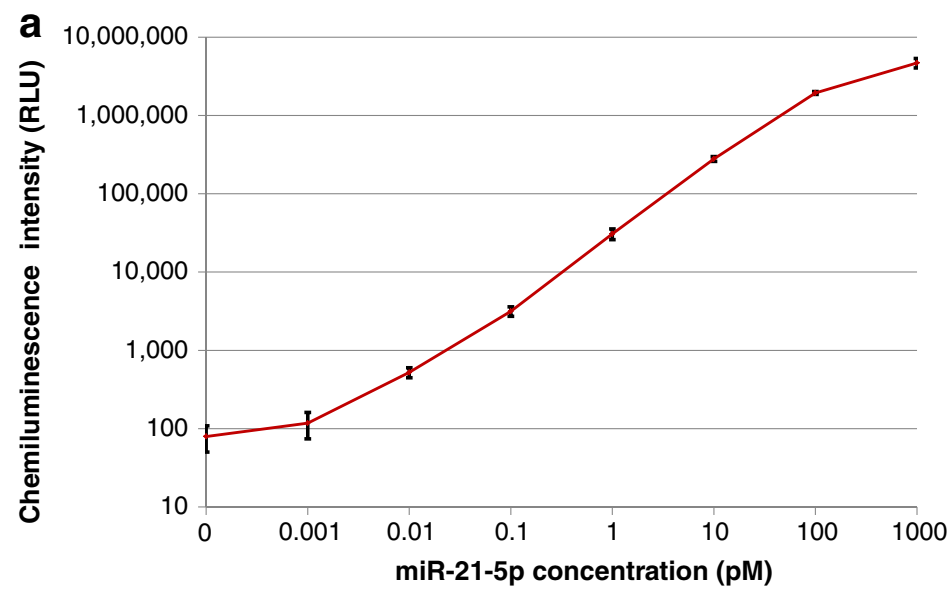

b
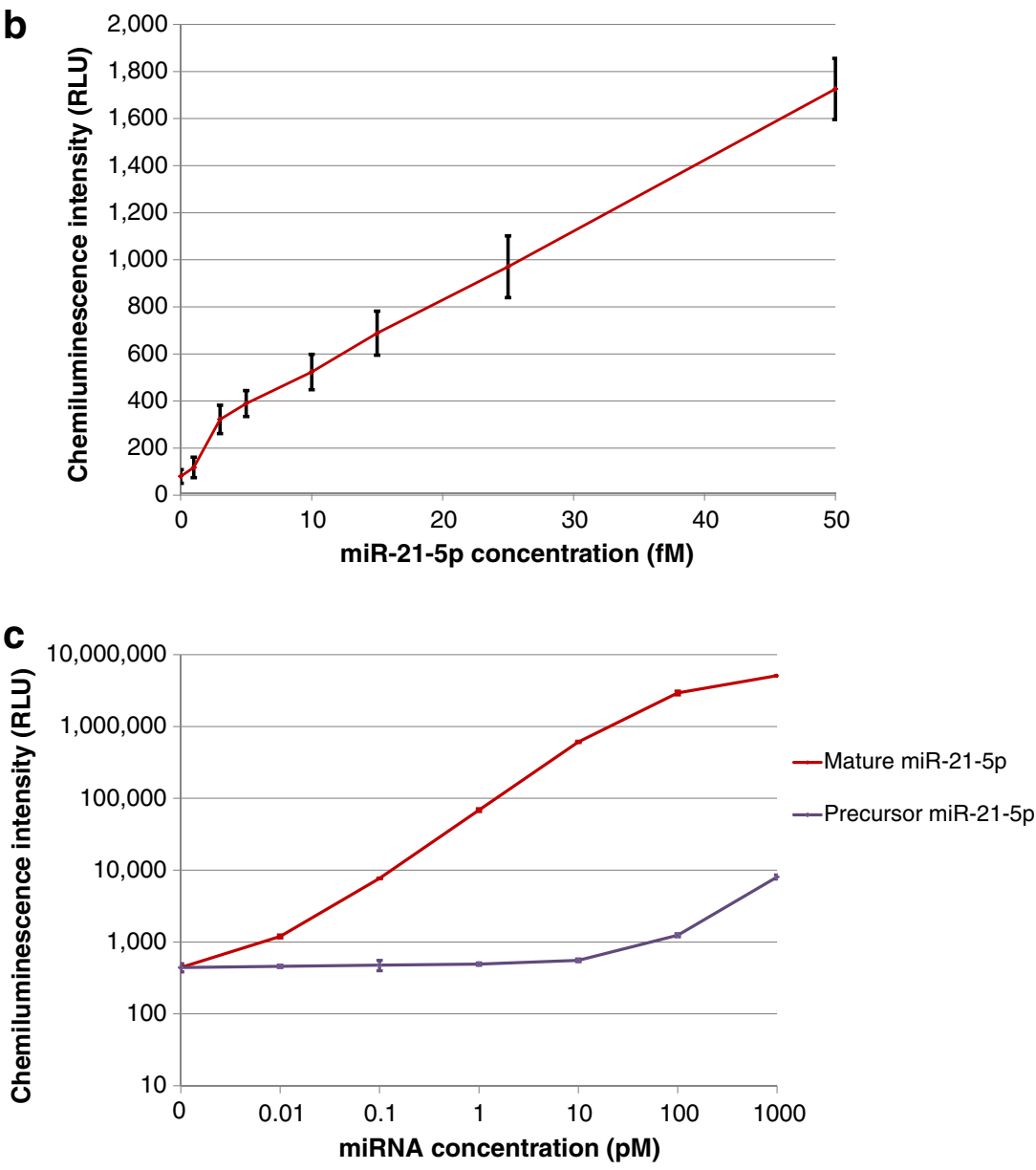

two-step amplification assay by layering linear amplification reactions [41]. We measured different concentrations of synthetic miR-21-5p from 0 to $1000 \mathrm{pM}$ in replicates of 5 on the analyzer. The obtained dose-response curve is shown in Fig. 4a. The curve fitting of the dose-response curve with fitted equation and correlation coefficient is shown in ESM Fig. S3A. For determining the precise detection limit, we further measured different concentrations of synthetic miR21-5p from 0 to $50 \mathrm{fM}$ in the samples in replicates of 20 (Fig. 4b). The sample with 0-fM (blank) and 3-fM miR-21-5p showed the mean CI of 79 relative light unit (RLU) (standard deviation (SD) 15) and 322 RLU (SD 30), respectively. The $3 \mathrm{fM}$ of miR-21-5p was distinguished from the blank sample ( $P$ (two-tailed unpaired $t$ test) $<10^{-21}$ ). The two-step amplification assay achieved the detection sensitivity value of $3 \mathrm{fM}$ for miR-21-5p, significantly lower than the cutoff concentrations in serum around $50 \mathrm{fM}$ [13]. This highly sensitive detection is attributed not only to the introduction 
Fig. 5 a Cross reactivity test of four miRNAs (miR-200b, miR200c, miR-141, miR-429) in miR-200 family in the two-step amplification assay for miR-200a on the analyzer. The bar shows \pm 2 standard deviations. Each sample was measured in triplicate except for the blank sample (rep. = 5). $\mathbf{b}$ Cross reactivity test of four miRNAs (miR-200a, miR-200c, miR-141, miR-429) in the twostep amplification assay for miR$200 \mathrm{~b}$ on the analyzer. The bar shows \pm 2 standard deviations. Each sample was measured in triplicate except for the blank sample (rep. = 5). c Cross reactivity test of four miRNAs (miR200a, miR-200b, miR-141, miR$429)$ in the two-step amplification assay for miR-200c on the analyzer. The bar shows \pm 2 standard deviations. Each sample was measured in triplicate except for the blank sample (rep. $=5$ )
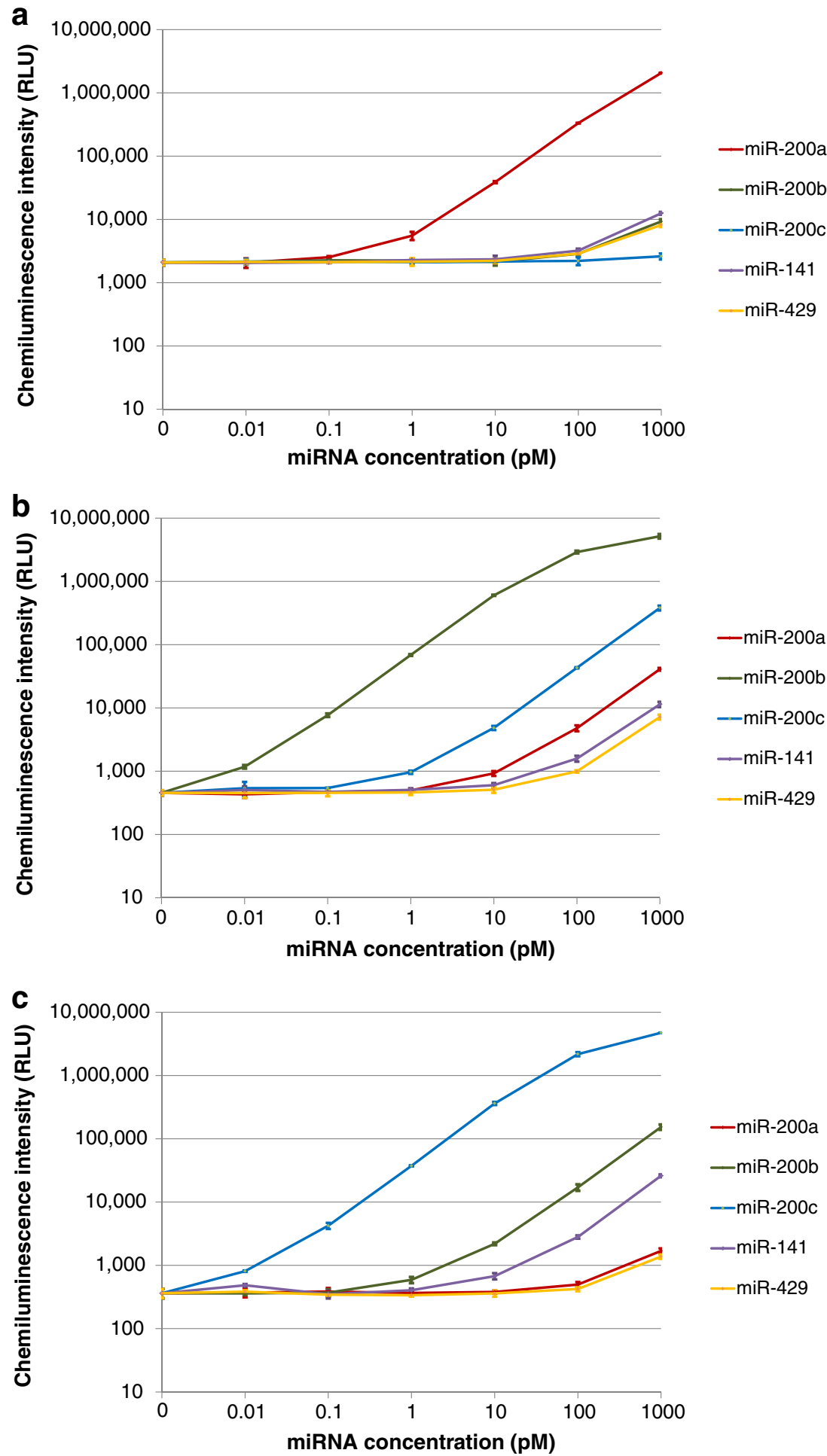

of cover sequence and layering linear amplification reactions, but also to the application of chemiluminescence reaction with Capture DNA probe and Chemiluminescence DNA probe emulating the common sandwich assay in immunoassay. This reaction design is suitable for the fully automated immunoassay analyzer.
In Fig. 4a, the CVs of CI for the target at concentrations from 0.01 to $1000 \mathrm{pM}$ were less than $8 \%$. The $\mathrm{CV}$ of CI even at low target concentration of $0.001 \mathrm{pM}$ and that of the background $\mathrm{CI}$ at $0 \mathrm{pM}$ were both $18 \%$. In Fig. $4 \mathrm{~b}$, the CVs of CI for the target at concentrations of $3 \mathrm{fM}$ were $9 \%$, and those at five concentrations from 5 to $50 \mathrm{fM}$ were less than $8 \%$. These 
Fig. 6 a Dose response on the spiked miD-21-5p, which was the single-stranded DNA with the same sequence as miR-21-5p, in human serum at the concentrations from 0 to $1000 \mathrm{pM}$ in the two-step amplification assay for miR-21-5p on the analyzer. The bar shows \pm 2 standard deviations. Each sample was measured in replicates of 10 except for the blank serum sample (rep. $=20)$. $\mathbf{b}$ Dose response for the detection sensitivity test on the spiked miD$21-5 \mathrm{p}$ in human serum at the concentrations from 0 to $100 \mathrm{fM}$ in the two-step amplification assay for miR-21-5p on the analyzer. The bar shows \pm 2 standard deviations. Each sample was measured in replicates of 10 except for the blank serum sample (rep. $=20$ )

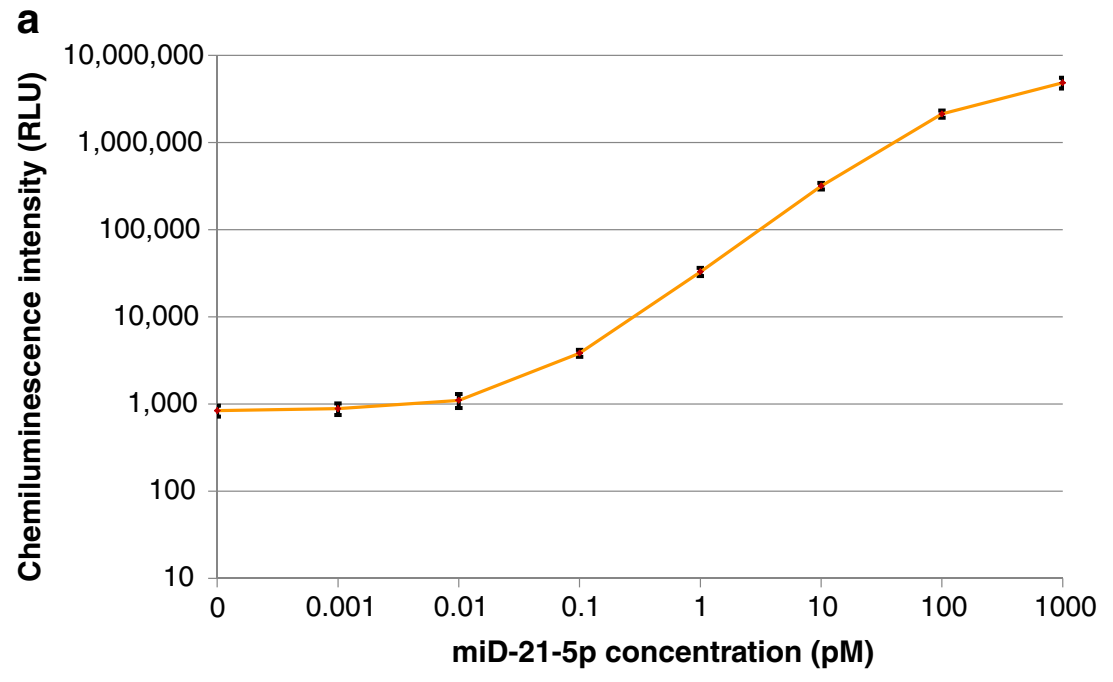

b

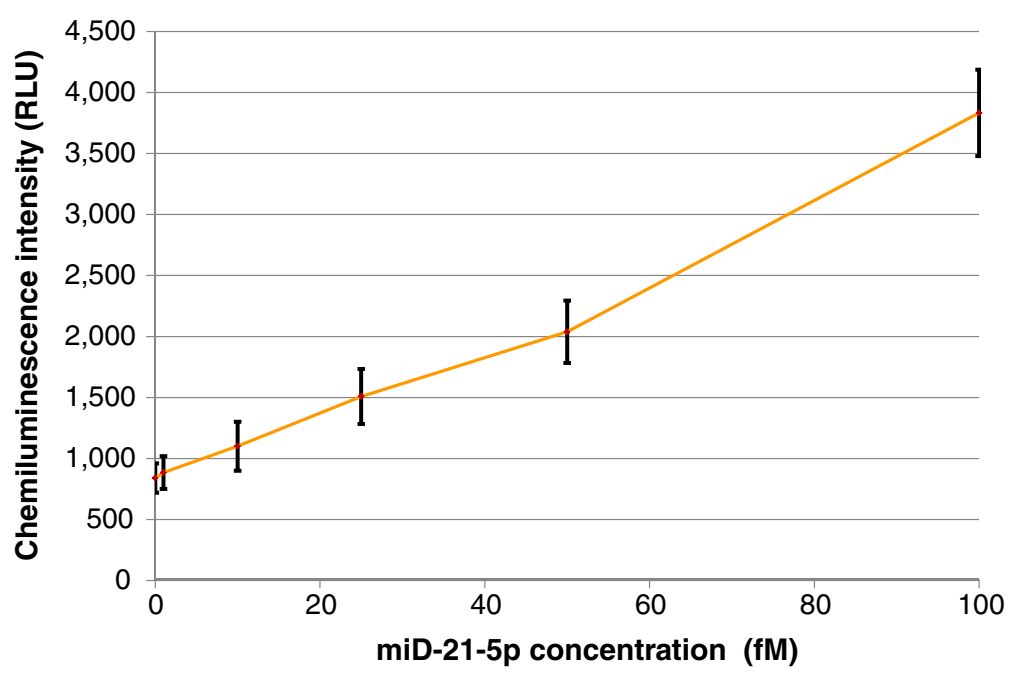

results indicated the high sensitivity and reproducibility of the assay.

Amplification rates as the ratios of amplified Signal DNA2 concentration to the target miR-21-5p concentration were estimated by using the calibration curve of Signal DNA2. The calibration curve of Signal DNA2 was generated in the measurement with serially diluted Signal DNA2 in $10 \mathrm{mM}$ Tris- $\mathrm{HCl}$, $0.01 \%$ BSA (pH 8.0) (ESM Fig. S3B, C). The mean amplification rate for the target concentrations from 0.1 to 10 pM was 103 fold. For comparison, we also estimated the amplification rate in the one-step amplification assay (ESM Fig. S4), based on the results shown in Fig. 3a. The obtained mean amplification rate for the target miR-21-5p at concentrations from 0.1 to $10 \mathrm{pM}$ was 26-fold. The cascading of signal DNA generation achieved the higher amplification rate in the two-step amplification assay for miR-21-5p than that in the one-step amplification assay.

We also investigated whether precursor miR-21-5p (60 nt), which contains mature miR-21-5p sequence and forms a secondary structure, and mature miR-21-5p (22 nt) were distinguished in the two-step amplification assay. The samples of synthetic precursor miR-21-5p and mature miR-21-5p at the concentrations from 0 to $1000 \mathrm{pM}$ were measured. The cross reactivity evaluated as the ratio of interfering mature miR-21$5 p$ concentration to precursor miR-21-5p concentration was, at the highest, $0.01 \%$ at the concentration of $1000 \mathrm{pM}$ (Fig. $4 c)$. The result indicated that the miR-21-5p assay with Converter DNA-21 preferably detected mature miR-21-5p than precursor miR-21-5p.

\section{Automated miR-200 family detection with two-step amplification on the analyzer}

The miR-200 family consists of miR-200a, miR-200b, miR200c, miR-141, and miR-429 which have highly homologous sequences (ESM Table S1B) [39]. We investigated the cross reactivity of the two-step amplification assays for miR-200a, 
miR-200b, and miR-200c by evaluating the ratio of interfering miRNA concentrations of each assay to each synthetic miRNA concentration. The samples of synthetic miRNAs, each containing miR-200a, miR-200b, miR-200c, miR-141, or miR-429 at the concentrations from 0 to $1000 \mathrm{pM}$, were measured in three respective assays with Converter DNAs having TBS for miR-200a, miR-200b, and miR-200c. In the assay for miR-200a, the cross reactivity values were not higher than $0.20,0.01,0.30$, and $0.19 \%$ for miR-200b, miR200c, miR-141, and miR-429, respectively (Fig. 5a). In the assay for miR-200b, the cross reactivity values were not higher than $0.06,0.64,0.02$, and $0.01 \%$ for miR-200a, miR200c, miR-141, and miR-429, respectively (Fig. 5b). In the assay for miR-200c, the cross reactivity values were 0.003 , $0.44,0.07$, and $0.002 \%$ for miR-200a, miR-200b, miR-141, and miR-429, respectively (Fig. $5 \mathrm{c}$ ). The similar miRNA sequences did not cause the false positive signal higher than $0.64 \%$, showing high sequence specificity of the two-step amplification assays for miR-200 family detection.

These results indicated that our miRNA assays with twostep amplification on the analyzer have high reproducibility and sequence specificity and could be clinically useful in cancer detection.

\section{Automated nucleic acid detection in human serum on the analyzer}

We performed the two-step amplification assay in normal human serum containing the ssDNA on the analyzer. MiD-21-5p spiked in serum samples at different concentrations from 0.001 to $1000 \mathrm{pM}$ and a blank at $0 \mathrm{pM}$ were measured in replicates of 10 and 20 , respectively, on the automated analyzer. The obtained dose-response curve is shown in Fig. 6a. The curve fitting of the dose-response curve with fitted equation and correlation coefficient is shown in ESM Fig. S5A.

To determine the sensitivity of the assay, we further measured different concentrations of miD-21-5p in serum samples from 1 to $100 \mathrm{fM}$ in replicates of 10 and a blank at $0 \mathrm{fM}$ in replicates of 20 (Fig. 6b). The mean CI calculated for the target miD-21-5p at the concentrations of 0 and $10 \mathrm{fM}$ were 840 RLU (SD 60) and 1101 RLU (SD 100), respectively. The $10 \mathrm{fM}$ of miD-21-5p in serum was distinguished from the blank serum sample ( $P$ (two-tailed unpaired $t$ test) $<10^{-8}$ ). The CVs of CI in the measurement with the target miD-21$5 \mathrm{p}$ in serum at concentrations from 0 to $1000 \mathrm{pM}$ were less than $8 \%$ except for $9 \%$ at $0.01 \mathrm{pM}$, which again represented the high reproducibility of the assay. The mean amplification rate for the target concentrations from 0.1 to $10 \mathrm{pM}$ was 126 fold (see ESM Fig. S5B, C). These results indicated that the two-step amplification assay for ssDNA in normal human serum had high detection sensitivity and reproducibility.

The physiological temperature condition of constant $37^{\circ} \mathrm{C}$ eliminated the need to purify the miRNA due to blood clotting caused by high-temperature conditions. Also, detergent addition, which is required for detaching miRNAs from the protein or breaking down exosomes in human serum [42], was implemented in the fully automated process.

\section{Conclusions}

The fully automated miRNA measurement was developed by adapting an isothermal DNA amplification reaction to the existing automated immunoassay analyzer, ARCHITECT i system. The reaction temperature at constant $37^{\circ} \mathrm{C}$ eliminates the purification process and results in a testing time of $44 \mathrm{~min}$ and a throughput of 66 tests per hour. The proposed assay for a cancer-related miRNA showed high detection sensitivity, high reproducibility, and low cross reactivity. Implementation of the measurement of miRNAs as potential cancer markers together with immunoassays for the conventional protein markers on the single automated analyzer would allow the precise diagnosis and reduce the cost of nucleic acid test. We believe our work will enable early cancer diagnosis based on high throughput measurement of multiple biomarkers in full automation, which is useful both for patients and clinical laboratories in terms of testing time, cost, and accurate diagnosis.

Acknowledgments This work was partially supported by JST and AMED Adaptable and Seamless Technology Transfer Program through Target-driven R\&D (A-STEP). The authors thank the research members at Abbott Japan and Tokyo Institute of Technology for their technical support in the research and Akiyoshi Fukamizu, Ph.D. (Life Science Center for Survival Dynamics, Tsukuba Advanced Research Alliance, University of Tsukuba) for critical discussions.

\section{Compliance with ethical standards}

Conflict of interest A part of this work by Ken Komiya was funded by Abbott Laboratories. Makoto Komori, Takuma Shirakawa, Takamitsu J. Morikawa, and Toru Yoshimura are employees of Abbott Japan which markets ARCHITECT i system. There are no other conflicts of interest.

Open Access This article is distributed under the terms of the Creative Commons Attribution 4.0 International License (http:// creativecommons.org/licenses/by/4.0/), which permits unrestricted use, distribution, and reproduction in any medium, provided you give appropriate credit to the original author(s) and the source, provide a link to the Creative Commons license, and indicate if changes were made.

\section{References}

1. Esteller M. Non-coding RNAs in human disease. Nat Rev Genet. 2011;12:861-74.

2. Pritchard CC, Cheng HH, Tewari M. MicroRNA profiling: approaches and considerations. Nat Rev Genet. 2012;13:358-69.

3. Cimmino A, Calin GA, Fabbri M, Iorio MV, Ferracin M, Shimizu M, et al. miR-15 and miR-16 induce apoptosis by targeting BCL2. Proc Natl Acad Sci USA. 2005;102:13944-9. 
4. Lu J, Getz G, Miska EA, Alvarez-Saavedra E, Lamb J, Peck D, et al. MicroRNA expression profiles classify human cancers. Nature. 2005;435:834-8.

5. Calin GA, Croce CM. MicroRNA signatures in human cancers. Nat Rev Cancer. 2006;6:857-66.

6. Rosenfeld N, Aharonov R, Meiri E, Rosenwald S, Spectori Y, Zepeniuk M, et al. MicroRNAs accurately identify cancer tissue origin. Nat Biotechnol. 2008;26:462-9.

7. Mitchell PS, Parkin RK, Kroh EM, Fritz BR, Wyman SK, Pogosova-Agadjanyan EL, et al. Circulating microRNAs as stable blood-based markers for cancer detection. Proc Natl Acad Sci USA. 2008;105:10513-8.

8. Arroyo JD, Chevillet JR, Kroh EM, Ruf IK, Pritchard CC, Gibson $\mathrm{DF}$, et al. Argonaute 2 complexes carry a population of circulating microRNAs independent of vesicles in human plasma. Proc Natl Acad Sci USA. 2011;108:5003-8.

9. Turchinovich A, Weiz L, Langheinz BB. Characterization of extracellular circulating microRNA. Nucleic Acids Res. 2011;39:722333.

10. Zhu H, Fan GC. Extracellular/circulating microRNAs and their potential role in cardiovascular disease. Am J Cardiovasc Dis. 2011;1:138-49.

11. Kosaka N, Iguchi H, Ochiya T. Circulating microRNA in body fluid: a new potential biomarker for cancer diagnosis and prognosis. Cancer Sci. 2010;101:2087-92.

12. Toiyama Y, Takahashi M, Hur K, Nagasaka T, Tanaka K, Inoue Y, et al. Serum miR-21 as a diagnostic and prognostic biomarker in colorectal cancer. J Natl Cancer Inst. 2013;105:849-59.

13. Zeng Z, Wang J, Zhao L, Hu P, Zhang H, Tang X, et al. Potential role of microRNA-21 in the diagnosis of gastric cancer: a metaanalysis. PloS One. 2013;8:e73278.

14. Morimura R, Komatsu S, Ichikawa D, Takeshita H, Tsujiura M, Nagata $\mathrm{H}$, et al. Novel diagnostic value of circulating miR-18a in plasma of patients with pancreatic cancer. Br J Cancer. 2011;105: 1733-40.

15. Faltejskova PV, Slaby O. Circulating blood-borne microRNAs as biomarkers in solid tumors. EXS. 2015;106:75-122.

16. He Y, Lin J, Kong D, Huang M, Xu C, Kim TK, et al. Current state of circulating MicroRNAs as cancer biomarkers. Clin Chem. 2015;61:1138-55.

17. Hong C, Baek A, Hah SS, Jung W, Kim DE. Fluorometric detection of microRNA using isothermal gene amplification and graphene oxide. Anal Chem. 2016;88:2999-3003.

18. Kalogianni DP, Kalligosfyri PM, Kyriakou IK, Christopoulos TK. Advances in microRNA analysis. Anal Bioanal Chem. 2018;410: 695-713.

19. D'Agata R, Spoto G. Advanced methods for microRNA biosensing: a problem-solving perspective. Anal Bioanal Chem. 2019. https://doi.org/10.1007/s00216-019-01621-8.

20. Liu Y, Zhang J, Tian J, Fan X, Geng H. Multiplex detection of microRNAs by combining molecular beacon probes with $\mathrm{T} 7$ exonuclease-assisted cyclic amplification reaction. Anal Bioanal Chem. 2017;409:107-14.

21. Clancy E, Burke M, Arabkari V, Barry T, Kelly H, Dwyer RM, et al. Amplification-free detection of microRNAs via a rapid microarraybased sandwich assay. Anal Bioanal Chem. 2017;409:3497-505.

22. Ach RA, Wang H, Curry B. Measuring microRNAs: comparisons of microarray and quantitativePCR measurements, and of different total RNA prep methods. BMC Biotechnol. 2008;8:69.

23. Navarro E, Serrano-Heras G, Castano MJ, Solera J. Real-time PCR detection chemistry. Clin Chim Acta. 2015;439:231-50.

24. Zhao Y, Chen F, Li Q, Wang L, Fan C. Isothermal amplification of nucleic acids. Chem Rev. 2015;115:12491-545.

25. Nolan T, Bustin SA. PCR technology: current innovations. 3rd Ed. CRC Press; 2013. p. 357.
26. Amendola A, Coen S, Belladonna S, Pulvirenti FR, Clemens JM, Capobianchi MR. Improving clinical laboratory efficiency: a timemotion evaluation of the Abbott m2000 RealTime and Roche COBAS AmpliPrep/COBAS TaqMan PCR systems for the simultanenous quantitation of HIV-1 RNA and HCV RNA. Clin Chem Lab Med. 2011;49:1283-8.

27. Kappel A, Backes C, Huang Y, Zafari S, Leidinger P, Meder B, et al. MicroRNA in vitro diagnostics using immunoassay analyzers. Clin Chem. 2015;61:600-7.

28. Kricka L, Wilson R. RNA testing now automated. Clin Chem. 2015;61:571-2.

29. Yoshimura T, Fujita K, Kinukawa H, Matsuoka Y, Patil R, Beligere $\mathrm{G}$, et al. Development and analytical performance evaluation of an automated chemiluminescent immunoassay for pro-gastrin releasing peptide (ProGRP). Clin Chem Lab Med. 2009;47:1557-63.

30. Ingen HE, Chan DW, Hubl W, Miyachi H, Molina R, Pitzel L, et al. Analytical and clinical evaluation of an electrochemiluminescence immunoassay for the determination of CA 125. Clin Chem. 1998;44:2530-6.

31. Slev PR, La'ulu SL, Roberts WL. Intermethod differences in results for total PSA, free PSA, and percentage of free PSA. Am J Clin Pathol. 2008;129:952-8.

32. Serdarevic N. The comparison between different immunoassays for serum carbohydrate antigen (CA19-9) concentration measurement. Acta Inform Med. 2018;26:235-9.

33. Notomi T, Mori Y, Tomita N, Kanda H. Loop-mediated isothermal amplification (LAMP): principle, features, and future prospects. J Microbiol. 2015;53:1-5.

34. Taylor SN, Van Der Pol B, Lillis R, Hook EW 3rd, Lebar W, Davis $\mathrm{T}$, et al. Clinical evaluation of 2 the BD ProbeTec ${ }^{\mathrm{TM}}$ Chlamydia trachomatis Qx amplified DNA assay on the BD Viper ${ }^{\mathrm{TM}}$ system with XTRTM technology. Sex Transm Dis. 2011;38:603-9.

35. Wu X, Zhu S, Huang P, Chen Y. Highly specific quantification of microRNA by coupling probe-rolling circle amplification and Forster resonance energy transfer. Anal Biochem. 2016;502:16-23.

36. Le BH, Seo YJ. Highly sensitive MicroRNA 146a detection using a gold nanoparticle-based CTG repeat probing system and isothermal amplification. Anal Chim Acta. 2018;999:155-60.

37. Komiya K, Komori M, Noda C, Kobayashi S, Yoshimura T, Yamamura M. Leak-free million-fold DNA amplification with locked nucleic acid and targeted hybridization in one pot. Org Biomol Chem. 2019. https://doi.org/10.1039/c9ob00521h.

38. Toiyama Y, Hur K, Tanaka K, Inoue Y, Kusunoki M, Boland R, et al. Serum miR-200c is a novel prognostic and metastasis-predictive biomarker in patients with colorectal cancer. Ann Surg. 2014;259: 735-43.

39. O'Brien SJ, Carter JV, Burton JF, Oxford BG, Schmidt MN, Hallion $\mathrm{JC}$, et al. The role of the miR-200 family in epithelial-mesenchymal transition in colorectal cancer: a systematic review. Int J Cancer. 2018;142:2501-11.

40. Kozomara A, Griffiths-Jones S. miRBase: annotating high confidence microRNAs using deep sequencing data. Nucleic Acids Res. 2014;42:D68-73.

41. Komiya K, Yamamura M. Cascading DNA generation reaction for controlling DNA nanomachines at a physiological temperature. $\mathrm{N}$ Gener Comput. 2015;33:213-29.

42. Yoo CE, Kim G, Kim M, Park D, Kang HJ, Lee M, et al. A direct extraction method for microRNAs from exosomes captured by immunoaffinity beads. Anal Biochem. 2012;431:96-8.

Publisher's note Springer Nature remains neutral with regard to jurisdictional claims in published maps and institutional affiliations. 

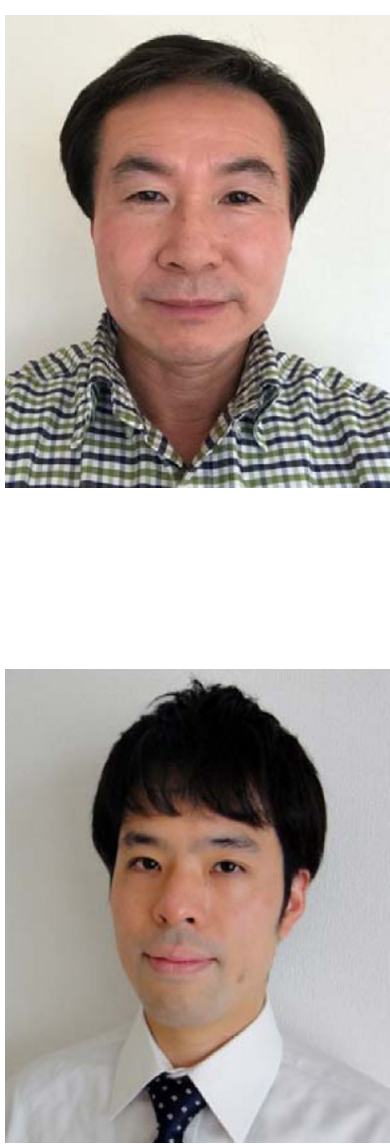

Ken Komiya is an assistant professor in the School of Computing at Tokyo Institute of Technology, who has been working towards the novel scientific discipline called "Molecular Robotics." His research interests include DNA-based Computing, Molecular Robotics, and Wet Artificial Life in addition to Analytical Chemistry utilizing DNA Nanotechnology.

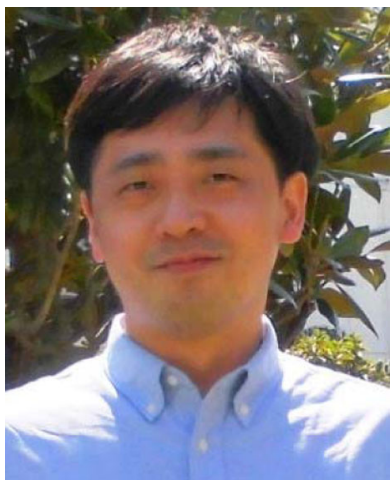

Takuma Shirakawa is a scientist in research and development, diagnostics division, Abbott Japan. His current work is focused on the immunoassay product design for the robust manufacturing process, and he is interested in the physical-chemical properties of excipient materials in immunoassay reagent and the complex interaction between reagent components, and biomarkers in clinical samples.

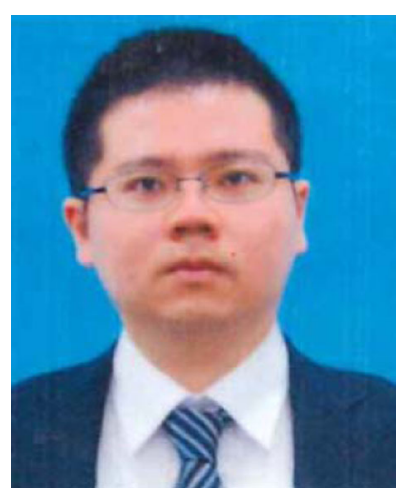

Takamitsu J. Morikawa is a scientist in the Research and Development of Diagnostics Division of Abbott Japan. He got a Ph.D. at Osaka University in 2015. His majors are Biophysics (single molecule imaging) and Molecular Biology (protein engineering, especially fluorescent protein).

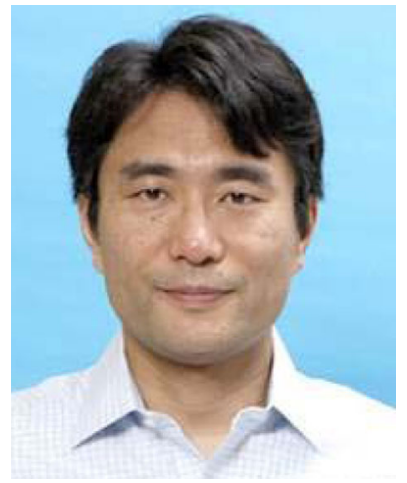

Toru Yoshimura is a director in the Research and Development of Diagnostics Division of Abbott Japan. He has been leading the projects of next-generation diagnostic technologies and discovery research of new diagnostic markers in addition to the product development of immunoassay for the detection of cancers and infectious diseases. He was appointed to a research fellow of Abbott Laboratories. 